Editorial

\title{
FGF-23 and left ventricular hypertrophy
}

\author{
Volume 9 Issue 3 - 2017
}

\section{Editorial}

Early detection of risk factors for enhanced primary prevention and novel therapies for treating the chronic consequences of cardiovascular disease (CVD) are of the utmost importance for reducing morbidity. Recently, fibroblast growth factors (FGFs) have been intensively studied as potential new molecules in the prevention and treatment of CVD mainly attributing to its metabolic effects and angiogenic actions. ${ }^{1}$ FGFs are multifunctional proteins; most commonly mitogens with regulatory, morphological and endocrine effects. They have been alternately referred to as "pluripotent" growth factors and as "promiscuous" growth factors due to their multiple actions on multiple cell types. ${ }^{2,3}$ Some members of the endocrine FGF family (FGFs FGF-1, 2, 4, 5, 19, 21 and 23) have shown multiple roles in regulating metabolic rate, adiposity, glucose homeostasis as well as direct effect on heart, influencing severity and extent of cardiovascular health. ${ }^{1}$ Among these FGFs, notably FGF-21 has shown cardioprotective effects such as improving lipoprotein profile, endothelial dysfunction and reducing plaque formation. ${ }^{1}$ FGF-23; member of the FGF19 subfamily shows the highest degree of homology with FGF-21., ${ }^{2,3}$ However, contrary to FGF-21, elevated levels of the bone-derived, phosphate-regulating hormone FGF-23 has emerged as a novel candidate predictive of cardiovascular events and death, independently of traditional and CKD-specific risk factors. ${ }^{1,2}$

FGF-23 was first identified in the ventrolateral thalamic nucleus of the mouse brain, ${ }^{4}$ and its importance was revealed in patients with autosomal dominant hypophosphatemic rickets (ADHR) $)^{5}$ wherein the missense mutation of the FGF-23 gene conferred resistance to the proteolytic cleavage of the FGF-23 protein. The full-length active FGF-23 increased phosphorus excretion leading to hypophosphatemia. FGF-23 is mainly expressed by osteocytes and osteoblasts in bone, but it is also expressed by salivary glands, stomach, and at much lower concentrations by other tissues, including skeletal muscle, brain, mammary gland, liver, and heart. ${ }^{6}$

FGF-23 maintains serum phosphate levels in the normal range by inhibiting $\mathrm{NaPi}-2 \mathrm{a}$ and $\mathrm{NaPi}-2 \mathrm{c}-\mathrm{co}$-transport on the brush border membrane of proximal tubules thereby suppressing renal phosphate absorption and promoting its excretion. ${ }^{7}$ In order to transmit its signal, FGF-23 forms a heterotrimer complex with receptor FGF1Rc and coreceptor Klotho (a-Klotho) -an anti-aging protein. Klotho binds to the C-terminal of FGF-23 which is cleaved during its functional dynamics to produce inactive $\mathrm{C}$-terminal and $\mathrm{N}$ - terminal fragments. ${ }^{8}$ Thus, in the bloodstream, apart from the C-terminal inactive fragment of FGF23; two distinct forms of the FGF-23 protein circulate: a full-length mature active intact form (227 amino acids) and a shorter form (155 amino acids-the $\mathrm{N}$-terminal fragment) lacking the unique 73 -amino acid $\mathrm{COOH}$ terminal tail. ${ }^{9}$ Thus, FGF-23 requires a transmembrane protein Klotho for its signaling. ${ }^{8}$ Klotho binds more avidly to FGFRs in the presence of Klotho, and triggers intracellular signaling pathways that mediate its biological action. ${ }^{10}$

Another important function of FGF-23 is to act as a counter regulatory hormone to vitamin D. It decreases synthesis of 1,25 $(\mathrm{OH}) 2 \mathrm{D}$ by suppressing 1-alpha hydroxylase enzyme and accelerates its degradation by activating 24 hydroxylase. ${ }^{11}$ In a classic negative

\author{
Kavita Shalia \\ Sir H.N. Medical Research Society, Sir H.N. Hospital and \\ Research Centre, India
}

Correspondence: Kavita K Shalia, Sr. Scientist, Sir H.N. Medical Research Society, Sir H.N. Hospital and Research Centre, Court House, L.T. Road, Mumbai 400 002, India, Tel 2267673883, Email shaliakavita@gmail.com

Received: July 20, 2017 | Published: July 20, 2017

endocrine feedback loop, 1,25(OH)2 D stimulates FGF-23 secretion, and FGF-23 lowers levels of $1,25(\mathrm{OH})_{2}$ D. ${ }^{12}$ The regulation of FGF23 transcription is controlled by a vitamin $\mathrm{D}$ response element in the FGF-23 promoter $^{13}$ such that some vitamin D activity is essential for FGF-23 production. This is supported by the finding of undetectable FGF-23 levels in vitamin D receptor-ablated mice, even after dietary phosphate loading, and in 1- $\alpha$ hydroxylase-ablated mice. ${ }^{14}$ Klotho and the vitamin D endocrine system also reciprocally regulate each other wherein Klotho decreases 1, 25(OH)2D synthesis in the kidney. It has also been reported that the Vitamin D receptor controls expression of the Klotho gene. 1,25(OH) 2 D stimulates both Klotho and FGF-23 and both FGF-23 as well as Klotho inhibit $1,25(\mathrm{OH})_{2} \mathrm{D}$ via inhibiting $1 \alpha$-hydroxylase. . $^{15,16}$

First evidence linking FGF-23 and cardiac disease was presented by Corda et al., ${ }^{17}$ and Scheinowitz et al., ${ }^{18}$ who reported that FGF-23 causes cardiac hypertrophy by inducing changes in gene expression that are similar to those caused by chronic pressure overload. ${ }^{17,18}$ This results in left ventricular dysfunction (LVD) that is characterized by increased extracellular matrix deposition, hypertrophy, and apoptosis of individual myocytes and increased risk of congestive heart failure and death. ${ }^{19}$ In an in vitro experimental system escalating doses of FGF-23 induced a dose-dependent increase in the surface area of neonatal rat ventricular cardiac myocytes and activated hypertrophic gene programs. ${ }^{20}$ FGFR inhibitors prevented FGF-23-mediated hypertrophy, indicating that the effect was mediated by FGFRs, which are expressed on cardiac myocytes. These results were confirmed in a series of experiments in rodents. ${ }^{20}$

Most relevant to $\mathrm{CKD}$, left ventricular hypertrophy $(\mathrm{LVH})$ in uremic rats that manifest markedly elevated FGF-23 levels was prevented by treatment with a pan-FGFR inhibitor despite persistence 
of equally severe untreated hypertension. These results confirmed the hypertension-independent effect of FGF-23 that was reported in the prospective analysis of normotensive CRIC participants. ${ }^{20} \mathrm{An}$ especially important finding was that Klotho was not detected in cardiac myocytes, indicating that FGF-23-mediated hypertrophy of cardiac myocytes occurred independent of membrane-bound Klotho. ${ }^{20}$ The finding of LVH in Klotho heterozygous mice that had moderately elevated FGF-23 levels (3-fold above normal), and more severe LVH in Klotho-ablated mice that had markedly elevated FGF-23 levels ( $>15$-fold above normal) excluded the possibility that the soluble form of Klotho is needed for LVH to develop elevated FGF-23 levels. These results established the concept of Klotho-independent, direct end-organ toxicity of FGF-23 and a potentially prominent role of FGF-23 in the pathogenesis of uremic cardiomyopathy. ${ }^{21}$ Thus, LVH is one plausible biological mechanism to explain the link between higher FGF-23 and greater risk of mortality.

In CKD, higher FGF-23 levels are demonstrated to be associated with $\mathrm{LVH}$, which is an important mechanism of congestive heart failure and arrhythmia, and a potent risk factor for mortality. ${ }^{22,23}$ Cross-sectional studies in CKD, ESRD and non-CKD populations demonstrated that elevated FGF-23 is independently associated with greater left ventricular mass index (LVMI) and greater prevalence of LVH. ${ }^{24,25}$ In the study conducted by Gutierrez et al., ${ }^{24}$ consisting of 162 patients with CKD who underwent echocardiograms and computed tomography scans to assess LVD; multivariable-adjusted regression analyses in the overall sample showed that increased log FGF-23 concentrations were independently associated with increased LVMI (5\% increase per $1 \mathrm{SD}$ increase in $\log \mathrm{FGF}-23, \mathrm{P}=0.01$ ), and risk of LVD (Odds Ratio OR per 1 SD increase in log FGF-23 2.1, 95\%CI 1.03, 4.2). Study Conducted by Mirza et al., ${ }^{26}$ suggests that elevated serum FGF-23 levels, even within the normal range were also associated with increased LVMI and increased risk for the presence of LVH in elderly subjects leading to cardiac complications. ${ }^{26}$ Thus the above pioneering echocardiographic studies reported an association between FGF-23 levels and LVH; in cohorts that comprised both CKD patients ${ }^{24}$ and individuals with intact renal function. ${ }^{26}$ Tonelli et al., ${ }^{27} 2005$ have reported FGF-23 as major predictors of mortality and potentially linked to a deranged $\mathrm{Ca}$-phosphate metabolism. ${ }^{27}$ In a study by Halankar et al., ${ }^{28}$ echocardiographic parameters were evaluated at recruitment and at six month in dialytic CKD patients wherein significant decrease in the EDV was observed at six month. EDV is the amount of blood in the ventricles just before systole and if it increases then eventually may cause LVH, pulmonary congestion and edema. Although this study did not reveal direct correlation of FGF-23 with LV function, at six month there was decrease observed in in iFGF-23, $\mathrm{P}$ and CaxP in dialytic CKD patients on regular calcium, vitamin $\mathrm{D}$ and phosphate binder treatment as well as significant decrease in EDV. Several other studies have reported strong associations between FGF-23 and cardiovascular risk factors such as old age, hypertension, obesity, smoking, diabetes and higher levels of inflammatory markers. ${ }^{24,-33}$ By demonstrating direct pathological effects of FGF23 on the heart, its mechanism and risk on CVD, future pharmacological interference with FGF23 and/or its cardiac receptor in CKD patients could be beneficial to prevent or treat CVD.

\section{Acknowledgments}

None.

\section{Conflicts of interest}

Author declares there are no conflicts of interest.

\section{Funding}

None.

\section{References}

1. Domouzoglou EM, Naka KK, Vlahos AP, et al. Fibroblast growth factors in cardiovascular disease: The emerging role of FGF21. Am J Physiol Heart Circ Physiol. 2015;309 (6): H1029-H1038.

2. Vlodavsky I, Korner G, Ishai-Michaeli R, et al. Extracellular matrixresident growth factors and enzymes: possible involvement in tumor metastasis and angiogenesis. Cancer Metastasis Rev. 1990;9(3):203-226.

3. Green PJ, Walsh FS, Doherty P. Promiscuity of fibroblast growth factor receptors. BioEssays. 1996;18(8):639-646.

4. Yamashita T, Yoshioka M, Itoh N. Identification of a novel fibroblast growth factor, FGF-23, preferentially expressed in the ventrolateral thalamic nucleus of the brain. Biochem Biophys Res Commun. 2000;277(2):494-498.

5. ADHR Consortium. Autosomal dominant hypophosphataemic rickets is associated with mutations in FGF23. Nat Genet. 2000;26(3):345-348.

6. Martin A, David V, Quarles LD. Regulation and function of the FGF23/ Klotho endocrine pathways. Physiol Rev. 2012;92(1):131-155.

7. Fukagawa M, Kazama JJ. With or without the kidney: the role of FGF23 in CKD. Nephrol Dial Transplant. 2005;20(7):1295-1298.

8. Kurosu H, Ogawa Y, Miyoshi M, et al. Regulation of fibroblast growth factor-23 signaling by Klotho. J Biol Chem . 2006;281(10):6120-6123.

9. Yamazaki Y, Okazaki R, Shibata M, et al. Increased circulatory level of biologically active full-length FGF-23 in patients with hypophosphatemic rickets/osteomalacia. J Clin Endocrinol Metab. 2002;87(11):4957-4960.

10. Urakawa I, Yamazaki Y, Shimada T, et al. Klotho converts canonical FGF receptor into a specific receptor for FGF23. Nature. 2006;444(7120):770-774.

11. Saito H, Kusano K, Kinosaki M, et al. Human fibroblast growth factor-23 mutants suppress $\mathrm{Na}+$-dependent phosphate co-transport activity and $1 \alpha, 25$-dihydroxyvitamin D3 production. $J$ Biol Chem. 2003;278(4):2206-2211.

12. Wolf M. Forging forward with 10 burning questions on FGF23 in kidney disease. J Am Soc Nephrol. 2010;21(9):1427-1435.

13. Liu S, Tang W, Zhou J, et al. Fibroblast growth factor 23 is a counterregulatory phosphaturic hormone for vitamin D. J Am Soc Nephrol. 2006;17(5):1305-1315.

14. Shimada T, Yamazaki Y, Takahashi M, et al. Vitamin D receptorindependent FGF-23 actions in regulating phosphate and vitamin D metabolism. Am J Physiol Renal Physiol . 2005;289(5):F1088-F1095.

15. Xu Y, Sun Z. Molecular basis of Klotho: from gene to function in aging. Endocr Rev. 2015;36(2):174-193.

16. Ding HY, Ma HX. Significant roles of anti-aging protein Klotho and fibroblast growth factor23 in cardiovascular disease. J Geriatr Cardiol. 2015;12(4):439-447.

17. Corda S, Mebazaa A, Gandolfini MP, et al. Trophic effect of human pericardial fluid on adult cardiac myocytes. Differential role of fibroblast growth factor-2 and factors related to ventricular hypertrophy. Circ Res. 1997; 81(5):679-687.

18. Scheinowitz M, Kotlyar A, Zimand S, et al. Basic fibroblast growth factor induces myocardial hypertrophy following acute infarction in rats. Exp Physiol. 1998;83(5):585-593.

19. Heineke J, Molkentin JD. Regulation of cardiac hypertrophy by intracellular signalling pathways. Nat Rev Mol Cell Biol. 2006;7(8):589-600. 
20. Faul C, Amaral AP, Oskouei B, et al. FGF23 induces left ventricular hypertrophy. J Clin Invest. 2011;121(11):4393-4408.

21. Wolf M. Update on fibroblast growth factor 23 in chronic kidney disease. Kidney Int. 2012; 82(7):737-747.

22. Silberberg JS, Barre PE, Prichard SS, et al. Impact of left ventricular hypertrophy on survival in end-stage renal disease. Kidney Int. 1989;36(2):286-290.

23. Glassock RJ, Pecoits-Filho R, Barberato SH. Left ventricular mass in chronic kidney disease and ESRD. Clin J Am Soc Nephrol. 2009; 4 (Suppl 1):S79-S91.

24. Gutierrez OM, Mannstadt M, Isakova T, et al. Fibroblast growth factor 23 and mortality among patients undergoing hemodialysis. $N$ Engl $J$ Med. 2008;359(6):584-592.

25. Seiler S, Cremers B, Rebling NM, et al. The phosphatonin fibroblast growth factor 23 links calcium-phosphate metabolism with left-ventricular dysfunction and atrial fibrillation. Eur Heart J. 2011;32(21):2688-2696.

26. Mirza MA, Larsson A, Melhus H, et al. Serum intact FGF23 associate with left ventricular mass, hypertrophy and geometry in an elderly population. Atherosclerosis. 2009; 207(2):546-551.

27. Tonelli M, Sacks F, Pfeffer M, et al. Relation between serum phosphate level and cardiovascular event rate in people with coronary disease. Circulation. 2005;112(17):2627-2633.
28. Halankar A, Sivaraman S, Shalia K. FGF-23 and Hyperphosphatemia in Dialysis Dependent Chronic Kidney Disease Patients. Urol Nephrol Open Access J . 2017;4(5):00141.

29. Isakova T, Xie H, Yang W, et al. Fibroblast growth factor 23 and risks of mortality and end-stage renal disease in patients with chronic kidney disease. JAMA. 2011; 305(23):2432-2439.

30. Kendrick J, Cheung AK, Kaufman JS, et al. FGF-23 associates with death, cardiovascular events, and initiation of chronic dialysis. J Am SOC Nephrol. 2011;22(10):1913-1922.

31. Parker BD, Schurgers LJ, Brandenburg VM, et al. The associations of fibroblast growth factor 23 and uncarboxylated matrix Gla protein with mortality in coronary artery disease: the Heart and Soul Study. Ann of Intern Med. 2010;152(10):640-648.

32. Manghat P, Fraser WD, Wierzbicki AS, et al. Fibroblast growth factor-23 is associated with C-reactive protein, serum phosphate and bone mineral density in chronic kidney disease. Osteoporos Int. 2010;21(11):1853-1861.

33. Tanaka H, Hamano T, Fujii N, et al. The impact of diabetes mellitus on vitamin D metabolism in predialysis patients. Bone. 2009;45(5):949-955. 